\title{
Existence and uniqueness of positive solutions to fractional boundary value problems with nonlinear boundary conditions
}

Nemat Nyamoradi ${ }^{1}$, Dumitru Baleanu $u^{2,3,4^{*}}$ and Ravi P Agarwal ${ }^{5,6}$

"Correspondence:

dumitru@cankaya.edu.tr

${ }^{2}$ Department of Chemical and

Materials Engineering, Faculty of Engineering, King Abdulaziz

University, P.O. Box 80204, Jeddah, 21589, Saudi Arabia

3 Department of Mathematics and Computer Sciences, Faculty of Art and Sciences, Cankaya University, Ankara, 06530, Turkey

Full list of author information is

available at the end of the article

\begin{abstract}
In this manuscript, we consider two problems of boundary value problems for a fractional differential equation. A fixed point theorem in partially ordered sets and a contraction mapping principle are applied to prove the existence of at least one positive solution for both fractional boundary value problems.

MSC: 47H10; 26A33; 34A08
\end{abstract}

Keywords: cone; contraction mapping principle; fixed point theorem; Riemann-Liouville fractional derivative

\section{Introduction}

Fractional calculus is the field of mathematical analysis, which deals with the investigation and applications of integrals and derivatives of an arbitrary order. In its turn, mathematical aspects of studies on fractional differential equations were discussed by many authors (see, for example, Refs. [1-10] and the references therein). Some recent results on fractional boundary value problems on a infinite interval can be found in [11-15] and the references therein. For example, Liu in [11] studied the following boundary value problem for the fractional differential equations:

$$
\left\{\begin{array}{l}
D_{0^{+}}^{\alpha} u(t)=f\left(t, v(t), D_{0^{+}}^{p} v(t)\right), \quad t \in(0, \infty), \\
D_{0^{+}}^{\beta} v(t)=g\left(t, u(t), D_{0^{+}}^{q} u(t)\right), \quad t \in(0, \infty), \\
a \lim _{t \rightarrow 0} t^{2-\alpha} u(t)-b \lim _{t \rightarrow 0} D_{0^{+}}^{\alpha-1} u(t)=u_{0}, \\
c \lim _{t \rightarrow 0} t^{2-\beta} v(t)-d \lim _{t \rightarrow 0} D_{0^{+}}^{\beta-1} v(t)=v_{0}, \\
\lim _{t \rightarrow \infty} D_{0^{+}}^{\alpha-1} u(t)=u_{1}, \quad \lim _{t \rightarrow \infty} D_{0^{+}}^{\beta-1} v(t)=v_{1}
\end{array}\right.
$$

by using the properties of Green's function of the corresponding problem and the Schauder fixed point theorem, where $\alpha, \beta \in(1,2), p \in(0, \beta), q \in(0, \alpha), u_{0}, v_{0}, u_{1}, v_{1} \in \mathbb{R}$, $f, g:(0, \infty) \times \mathbb{R}^{2} \rightarrow \mathbb{R}$ are continuous functions, and $f, g$ may be singular at $t=0$.

(c) 2013 Nyamoradi et al.: licensee Springer. This is an Open Access article distributed under the terms of the Creative Commons Attribution License (http://creativecommons.org/licenses/by/2.0), which permits unrestricted use, distribution, and reproduction in any medium, provided the original work is properly cited. 
Liu et al. [12] considered the following boundary value problem for fractional differential equations:

$$
\left\{\begin{array}{l}
D_{0^{+}}^{\alpha} u(t)=f\left(t, v(t), D_{0^{+}}^{p} v(t)\right), \quad t \in(0, \infty), \\
D_{0^{+}}^{\beta} v(t)=g\left(t, u(t), D_{0^{+}}^{q} u(t)\right), \quad t \in(0, \infty), \\
\lim _{t \rightarrow 0} t^{2-\alpha} u(t)=u_{0}, \quad \lim _{t \rightarrow 0} t^{2-\beta} v(t)=v_{0}, \\
\lim _{t \rightarrow 0} D_{0^{+}}^{\alpha-1} u(t)=u_{1}, \quad \lim _{t \rightarrow 0} D_{0^{+}}^{\beta-1} v(t)=v_{1},
\end{array}\right.
$$

where $a, b, c, d>0, \alpha, \beta \in(1,2), p \in(\beta-1, \beta), q \in(\alpha-1, \alpha), u_{0}, v_{0}, u_{1}, v_{1} \in \mathbb{R}, f, g:(0, \infty) \times$ $\mathbb{R}^{2} \rightarrow \mathbb{R}$ are continuous functions, and $f, g$ may be singular at $t=0$. By using the properties of Green's function together with the Schauder fixed point theorem, it has been proved that this problem has at least one positive solution.

Our purpose in the first part of this paper is to show the existence of at least one positive solution for the following fractional problem:

$$
\left\{\begin{array}{l}
D_{0^{+}}^{\alpha} u(t)=f(t, u(t)), \quad t \in(0, \infty), \\
\lim _{t \rightarrow 0} t^{2-\alpha} u(t)=a, \quad \lim _{t \rightarrow 0} D_{0^{+}}^{\alpha-1} u(t)=b,
\end{array}\right.
$$

where $1<\alpha \leq 2, a, b \in \mathbb{R}, D_{0^{+}}^{\alpha}$ is the Riemann-Liouville fractional derivative of order $\alpha, f:(0, \infty) \times \mathbb{R} \rightarrow \mathbb{R}$ is a continuous function, and $f$ may be singular at $t=0$, i.e., $\lim _{t \rightarrow 0^{+}} f(t, \cdot)=+\infty$.

In the second part of this paper, we consider an infinite fractional boundary value problem for singular integro-differential equation of mixed type on the half line:

$$
\left\{\begin{array}{l}
D_{0^{+}}^{\alpha} u(t)=f(t, u(t),(S u)(t),(H u)(t)), \quad t \in(0, \infty), \\
\lim _{t \rightarrow 0} t^{2-\alpha} u(t)=a, \quad \lim _{t \rightarrow 0} D_{0^{+}}^{\alpha-1} u(t)=b
\end{array}\right.
$$

where $S, H:(0, \infty) \times(0, \infty) \rightarrow[0, \infty)$,

$$
(S u)(t)=\int_{0}^{t} K(t, s) u(s) d s, \quad(H u)(t)=\int_{0}^{\infty} H(t, s) u(s) d s
$$

The rest of the article is organized as follows: in Section 2, we shall recall certain results from the theory of the continuous fractional calculus. In Section 3, we shall provide some conditions, under which problem (1) has at least one positive solution. In Section 4, by suitable conditions, we will prove that problem (2) has at least one positive solution. Finally, in Section 5, we shall provide two numerical examples, which shall explicate the applicability of our results.

\section{Preliminaries}

In this section, we present some notations and preliminary lemmas that will be used in the proofs of the main results.

Definition 1 Let $X$ be a real Banach space. A non-empty closed set $P \subset X$ is called a cone of $X$ if it satisfies the following conditions:

(1) $x \in P, \mu \geq 0$, implies $\mu x \in P$, and

(2) $x \in P,-x \in P$, implies $x=0$. 
Definition $2[8,10]$ The Riemann-Liouville fractional integral operator of order $\alpha>0$ of function $f \in L^{1}\left(\mathbb{R}^{+}\right)$is defined as

$$
I_{0^{+}}^{\alpha} f(t)=\frac{1}{\Gamma(\alpha)} \int_{0}^{t}(t-s)^{\alpha-1} f(s) d s
$$

where $\Gamma(\cdot)$ is the Euler gamma function.

Definition $3[8,10]$ The Riemann-Liouville fractional derivative of order $\alpha>0$ of a continuous function $f:(0, \infty) \rightarrow \mathbb{R}$ is defined as

$$
D_{0^{+}}^{\alpha} f(t)=\frac{1}{\Gamma(n-\alpha)}\left(\frac{d}{d t}\right)^{n} \int_{0}^{t}(t-s)^{n-\alpha-1} f(s) d s
$$

where $n=[\alpha]+1$.

Lemma 1 [9] The equality $D_{0^{+}}^{\gamma} I_{0^{+}}^{\gamma} f(t)=f(t), \gamma>0$ holds for $f \in L^{1}(0,1)$.

Lemma 2 [9] Let $\alpha>0$, then the differential equation

$$
D_{0^{+}}^{\alpha} u=0
$$

has a unique solution $u(t)=c_{1} t^{\alpha-1}+c_{2} t^{\alpha-2}+\cdots+c_{n} t^{\alpha-n}, c_{i} \in \mathbb{R}, i=1, \ldots, n$, where $n-1<$ $\alpha \leq n$.

Lemma 3 [9] Let $\alpha>0$, then the following equality holds for $u \in L^{1}(0,1), D_{0^{+}}^{\alpha} u \in L^{1}(0,1)$;

$$
I_{0^{+}}^{\alpha} D_{0^{+}}^{\alpha} u(t)=u(t)+c_{1} t^{\alpha-1}+c_{2} t^{\alpha-2}+\cdots+c_{n} t^{\alpha-n},
$$

$c_{i} \in \mathbb{R}, i=1, \ldots, n$, where $n-1<\alpha \leq n$.

\section{Existence solution of problem (1)}

In this section, we study the existence and uniqueness of solutions of (1). To prove the main result, we need the following definitions and a preliminary lemma.

Let $C(0, \infty)$ be the set of all continuous functions on $(0, \infty)$. Choose $\sigma>-1$ and

$$
X:=\left\{u \in C(0, \infty): \frac{t^{2-\alpha}}{1+t^{\sigma+2}} u(t) \text { is bounded on }(0, \infty)\right\} .
$$

For $u \in X$, define the norm by

$$
\|u\|_{X}:=\sup _{t \in(0, \infty)}\left(\frac{t^{2-\alpha}}{1+t^{\sigma+2}}|u(t)|\right) .
$$

It is easy to show that $X$ is a real Banach space. We note that this Banach space can be equipped with a partial order given by

$$
x, y \in X, \quad x \leq y \quad \Leftrightarrow \quad x(t) \leq y(t) \quad \text { for all } t \in(0, \infty) .
$$

Define the classic metric given by

$$
d(x, y)=\sup _{t \in(0, \infty)}\left(\frac{t^{2-\alpha}}{1+t^{\sigma+2}}|x(t)-y(t)|\right)
$$


and the cone $P \subset X$ by

$$
P=\{u \in X: u(t) \geq 0, t \in(0, \infty)\} .
$$

Definition $4 f:(0, \infty) \times \mathbb{R} \rightarrow \mathbb{R}$ is called an $\alpha$-Caratheodory function if it satisfies the following assumptions:

(i) $t \rightarrow f\left(t, \frac{1+t^{\sigma+2}}{t^{2-\alpha}} u\right)$ is measurable on $(0, \infty)$ for every $u \in \mathbb{R}$,

(ii) $u \rightarrow f\left(t, \frac{1+t^{\sigma+2}}{t^{2-\alpha}} u\right)$ is continuous on $[0,+\infty)$ for all $t \in(0, \infty)$,

(iii) for each $r>0$, there exist $M_{r}>0$ and $\mu \in(-1, \sigma)$ such that

$$
\left|f\left(t, \frac{1+t^{\sigma+2}}{t^{2-\alpha}} u\right)\right| \leq M_{r} t^{\mu}, \quad t \in(0, \infty),|u| \leq r
$$

The following two lemmas are fundamental in the proofs of our main results.

Lemma $4[16]$ Let $(X, \leq)$ be a partially ordered set, and suppose that there exists a metric space. Assume that $X$ satisfies the following condition: if $\left\{x_{n}\right\}$ is a nondecreasing sequence in $X$ such that $x_{n} \rightarrow x$ then $x_{n} \leq x$ for all $n \in \mathbb{N}$. Let $T: X \rightarrow X$ be a nondecreasing mapping such that

$$
d(T x, T y) \leq d(x, y)-\varphi(d(x, y)), \quad \text { for all } x \geq y,
$$

where $\varphi:[0,+\infty) \rightarrow[0,+\infty)$ is a continuous and nondecreasing function such that is positive in $(0,+\infty)$ and $\varphi(0)=0$. If there exists $x_{0} \in X$ with $x_{0} \leq T\left(x_{0}\right)$, then $T$ has a fixed point.

If we consider that $(X, \leq)$ satisfies the following condition:

for $x, y \in X$, there exists $z \in X$ which is comparable to $x$ and $y$,

then we have the following lemma in [16].

Lemma 5 [16] Adding condition (6) to the hypotheses of Lemma 4, one obtains uniqueness of the fixed point of $T$.

Lemma 6 (See Lemma 2.1 in [11]) Suppose that $h:(0, \infty) \rightarrow \mathbb{R}$ is a given function satisfying that there exist numbers $M>0$ and $k \in(-1, \sigma)$ such that $|h(t)| \leq M t^{k}$ and $1<\alpha<2$, $a, b \in \mathbb{R}$. Then $u$ is a solution of

$$
\left\{\begin{array}{l}
D_{0^{+}}^{\alpha} u(t)=h(t), \quad t \in(0, \infty), \\
\lim _{t \rightarrow 0} t^{2-\alpha} u(t)=a, \quad \lim _{t \rightarrow 0} D_{0^{+}}^{\alpha-1} u(t)=b,
\end{array}\right.
$$

if and only if $u \in X$ and

$$
u(t)=\frac{1}{\Gamma(\alpha)} \int_{0}^{t}(t-s)^{\alpha-1} h(s) d s+\frac{b}{\Gamma(\alpha)} t^{\alpha-1}+a t^{\alpha-2} .
$$


Lemma 7 Let $-1<\sigma<1,1<\alpha<2, f:(0, \infty) \times[0,+\infty) \rightarrow[0,+\infty)$ is an $\alpha$-Caratheodory function, $t^{\sigma} f(t, u) \geq 0$ for $(t, u) \in(0, \infty) \times[0,+\infty)$ and $\lim _{t \rightarrow 0^{+}} f(t, \cdot)=\infty$. Assume that $t^{\sigma} f(t, \cdot)$ is a bounded function on $(0, \infty)$, and define the Hammerstein integral operator $T: P \rightarrow X$ by

$$
T u(t)=\frac{1}{\Gamma(\alpha)} \int_{0}^{t}(t-s)^{\alpha-1} f(s, u(s)) d s+\frac{b}{\Gamma(\alpha)} t^{\alpha-1}+a t^{\alpha-2}
$$

Then $T P \subset P$.

Proof The proof is straightforward, so we omit it here.

We state our main result as follows.

Theorem 1 Assume that $0<\sigma_{0}<1$, and the hypotheses of Lemma 7 hold. Suppose that

$$
0<\lambda \leq \frac{\Gamma(\alpha)}{\sup _{t \in(0, \infty)} \frac{t^{2-\sigma}}{1+t^{\sigma+2}} B\left(\alpha, 1-\sigma_{0}\right)}
$$

such that for $u, v \in[0,+\infty)$ with $u \geq v$ and $t \in(0, \infty)$,

$$
0 \leq t^{\sigma_{0}}\left[f\left(t, \frac{1+t^{\sigma+2}}{t^{2-\alpha}} u\right)-f\left(t, \frac{1+t^{\sigma+2}}{t^{2-\alpha}} v\right)\right] \leq \lambda \phi(u-v)
$$

where $\phi:[0,+\infty) \rightarrow[0,+\infty)$ is continuous and nondecreasing, $\varphi(u)=u-\phi(u)$ satisfies

(a) $\varphi:[0,+\infty) \rightarrow[0,+\infty)$ and is nondecreasing,

(b) $\varphi(0)=0$,

(c) $\varphi$ is positive in $(0,+\infty)$.

Then the boundary value problem (1) has a unique positive solution.

Proof Firstly, we claim that the operator $T$ is nondecreasing. To this end, by hypothesis, for $u \geq v$,

$$
\begin{aligned}
(T u)(t) & =\frac{1}{\Gamma(\alpha)} \int_{0}^{t}(t-s)^{\alpha-1} f(s, u(s)) d s+\frac{b}{\Gamma(\alpha)} t^{\alpha-1}+a t^{\alpha-2} \\
& =\frac{1}{\Gamma(\alpha)} \int_{0}^{t}(t-s)^{\alpha-1} s^{-\sigma_{0}} s^{\sigma_{0}} f(s, u(s)) d s+\frac{b}{\Gamma(\alpha)} t^{\alpha-1}+a t^{\alpha-2} \\
& \geq \frac{1}{\Gamma(\alpha)} \int_{0}^{t}(t-s)^{\alpha-1} s^{-\sigma_{0}} s^{\sigma_{0}} f(s, v(s)) d s+\frac{b}{\Gamma(\alpha)} t^{\alpha-1}+a t^{\alpha-2} \\
& =\frac{1}{\Gamma(\alpha)} \int_{0}^{t}(t-s)^{\alpha-1} f(s, v(s)) d s+\frac{b}{\Gamma(\alpha)} t^{\alpha-1}+a t^{\alpha-2}=(T v)(t) .
\end{aligned}
$$

Also, for $u \geq v$, by (4), one can get

$$
\begin{aligned}
d(T u, T v) & =\sup _{t \in(0, \infty)}\left(\frac{t^{2-\alpha}}{1+t^{\sigma+2}}|(T u)(t)-(T v)(t)|\right) \\
& =\sup _{t \in(0, \infty)} \frac{t^{2-\alpha}}{1+t^{\sigma+2}}\left[\frac{1}{\Gamma(\alpha)} \int_{0}^{t}(t-s)^{\alpha-1}(f(s, u(s))-f(s, v(s))) d s\right]
\end{aligned}
$$




$$
\begin{aligned}
= & \sup _{t \in(0, \infty)} \frac{t^{2-\alpha}}{1+t^{\sigma+2}}\left[\frac { 1 } { \Gamma ( \alpha ) } \int _ { 0 } ^ { t } ( t - s ) ^ { \alpha - 1 } \left(f\left(s, \frac{1+s^{\sigma+2}}{s^{2-\alpha}} \cdot \frac{s^{2-\alpha}}{1+s^{\sigma+2}} u(s)\right)\right.\right. \\
& \left.\left.-f\left(s, \frac{1+s^{\sigma+2}}{s^{2-\alpha}} \cdot \frac{s^{2-\alpha}}{1+s^{\sigma+2}} v(s)\right)\right) d s\right] \\
= & \sup _{t \in(0, \infty)} \frac{t^{2-\alpha}}{1+t^{\sigma+2}}\left[\frac { 1 } { \Gamma ( \alpha ) } \int _ { 0 } ^ { t } ( t - s ) ^ { \alpha - 1 } s ^ { - \sigma _ { 0 } } s ^ { \sigma 0 } \left(f\left(s, \frac{1+s^{\sigma+2}}{s^{2-\alpha}} \cdot \frac{s^{2-\alpha}}{1+s^{\sigma+2}} u(s)\right)\right.\right. \\
& \left.\left.-f\left(s, \frac{1+s^{\sigma+2}}{s^{2-\alpha}} \cdot \frac{s^{2-\alpha}}{1+s^{\sigma+2}} v(s)\right)\right) d s\right] \\
\leq & \sup _{t \in(0, \infty)} \frac{t^{2-\alpha}}{1+t^{\sigma+2}}\left[\frac{1}{\Gamma(\alpha)} \int_{0}^{t}(t-s)^{\alpha-1} s^{-\sigma_{0}} \lambda \phi\left(\frac{s^{2-\alpha}}{1+s^{\sigma+2}}(u(s)-v(s))\right) d s\right] .
\end{aligned}
$$

As the function $\phi(u)$ is nondecreasing, then for $u \geq v$,

$$
\phi\left(\frac{s^{2-\alpha}}{1+s^{\sigma+2}}(u(s)-v(s))\right) \leq \phi\left(\|u-v\|_{X}\right),
$$

and by the inequality above, we get

$$
\begin{aligned}
d(T u, T v) & \leq \sup _{t \in(0, \infty)} \frac{t^{2-\alpha}}{1+t^{\sigma+2}}\left[\frac{1}{\Gamma(\alpha)} \int_{0}^{t}(t-s)^{\alpha-1} s^{-\sigma_{0}} \lambda \phi\left(\frac{s^{2-\alpha}}{1+s^{\sigma+2}}(u(s)-v(s))\right) d s\right] \\
& \leq \lambda \phi\left(\|u-v\|_{X}\right) \sup _{t \in(0, \infty)} \frac{t^{2-\alpha}}{1+t^{\sigma+2}}\left[\frac{1}{\Gamma(\alpha)} \int_{0}^{t}(t-s)^{\alpha-1} s^{-\sigma_{0}} d s\right] \\
& \leq \lambda \phi\left(\|u-v\|_{X}\right) \sup _{t \in(0, \infty)} \frac{t^{2-\sigma_{0}}}{1+t^{\sigma+2}} \frac{1}{\Gamma(\alpha)} \int_{0}^{1}(1-\omega)^{\alpha-1} \omega^{-\sigma_{0}} d \omega \\
& =\lambda \phi\left(\|u-v\|_{X}\right) \frac{1}{\Gamma(\alpha)} B\left(\alpha, 1-\sigma_{0}\right) \sup _{t \in(0, \infty)} \frac{t^{2-\sigma_{0}}}{1+t^{\sigma+2}} \\
& \leq \phi\left(\|u-v\|_{X}\right)=\|u-v\|_{X}-\left(\|u-v\|_{X}-\phi\left(\|u-v\|_{X}\right)\right) .
\end{aligned}
$$

Suppose that $\varphi(u)=u-\phi(u)$ and $\varphi:[0,+\infty) \rightarrow[0,+\infty)$ is continuous, nondecreasing, positive in $(0,+\infty)$ and $\varphi(0)=0$. Thus, for $u \geq v, d(T u, T v) \leq d(u, v)-\varphi(d(u, v))$. Finally, take into account that for the zero function, $T 0 \geq 0$, by Lemma 4 , the boundary value problem $(1)$ has at least one positive solution. Moreover, this solution is unique since $(P, \leq)$ satisfies condition (6) and Lemma 5. This completes the proof.

Remark 1 Theorem 1 extends the result in [17] on the existence of a unique nonnegative solution for the following problem:

$$
\left\{\begin{array}{l}
D_{0^{+}}^{\alpha} u(t)+f(t, u(t))=0, \quad t \in(0,1) \\
u(0)=u^{\prime}(1)=u^{\prime \prime}(1)=0
\end{array}\right.
$$

Here the authors worked in the space $C[0,1]$.

\section{Existence solution of problem (2)}

In this section, we study the existence and uniqueness of solutions of (2). To prove the main result, we need the following assumptions: 
(H1) $k_{0}:=\sup _{t \in(0, \infty)} \frac{t^{2-\alpha}}{1+t^{\sigma+2}} \int_{0}^{t} \frac{1+s^{\sigma+2}}{s^{2-\alpha}} K(t, s) d s<\infty$ and $h_{0}:=\sup _{t \in(0, \infty)} \frac{t^{2-\alpha}}{1+t^{\sigma+2}} \int_{0}^{\infty} H(t, s) \frac{1+s^{\sigma+2}}{s^{2-\alpha}} d s<\infty ;$

(H2) There exist positive functions $L_{i}(t), i=1,2,3$ such that

$$
\begin{aligned}
& \| f\left(t, \frac{1+t^{\sigma+2}}{t^{2-\alpha}} u(t),\left(S \frac{1+t^{\sigma+2}}{t^{2-\alpha}} u\right)(t),\left(H \frac{1+t^{\sigma+2}}{t^{2-\alpha}} u\right)(t)\right) \\
& \quad-f\left(t, \frac{1+t^{\sigma+2}}{t^{2-\alpha}} v(t),\left(S \frac{1+t^{\sigma+2}}{t^{2-\alpha}} v\right)(t),\left(H \frac{1+t^{\sigma+2}}{t^{2-\alpha}} v\right)(t)\right) \| \\
& \leq L_{1}(t)|u-v|+L_{2}(t)|S u-S v|+L_{3}(t)|H u-H v|, \quad \forall u, v \in \mathbb{R}, t \in(0, \infty) ;
\end{aligned}
$$

(H3) There exists a number $v$ such that $\lambda_{0} \leq v<1, t \in(0, \infty)$, where

$$
\lambda_{0}=\left(1+k_{0}+h_{0}\right) I_{L}^{\alpha},
$$

and

$$
I_{L}^{\alpha}=\max \left\{\sup _{t \in(0, \infty)} \frac{t^{2-\alpha}}{1+t^{\sigma+2}} I_{0^{+}}^{\alpha} L_{i}(t), i=1,2,3\right\} .
$$

Theorem 2 Assume that (H1) holds, and $:(0, \infty) \times \mathbb{R} \times \mathbb{R} \times \mathbb{R} \rightarrow \mathbb{R}$ is a jointly continuous function, which satisfies (H2) that there exist numbers $M>0$ and $k \in(-1, \sigma)$ such that $|f(t, u(t),(S u)(t),(H u)(t))| \leq M t^{k}$. Then problem (2) has a unique solution, provided $\lambda_{0}<1$, where $\lambda_{0}$ is given in $(\mathrm{H} 3)$.

Proof Let the operator $L: X \rightarrow X$ be defined by the formula

$$
\begin{aligned}
L u(t)= & \frac{1}{\Gamma(\alpha)} \int_{0}^{t}(t-s)^{\alpha-1} f(s, u(s),(S u)(s),(H u)(s)) d s \\
& +\frac{b}{\Gamma(\alpha)} t^{\alpha-1}+a t^{\alpha-2}, \quad t \in(0, \infty) .
\end{aligned}
$$

Setting $\sup _{t \in(0, \infty)}\|f(t, 0,0,0)\|=M, \gamma_{1}=\frac{1}{\Gamma(\alpha+1)} \sup _{t \in(0, \infty)} \frac{t^{2}}{1+t^{\sigma+2}}, A_{1}=\sup _{t \in(0, \infty)} \frac{t}{1+t^{\sigma+2}}, A_{2}=$ $\sup _{t \in(0, \infty)} \frac{1}{1+t^{\sigma+2}}$ and choosing

$$
R \geq \frac{1}{1-v}\left|\left[\gamma_{1} M+\frac{b}{\Gamma(\alpha)} A_{1}+a A_{2}\right]\right|
$$

where $\lambda_{0} \leq v<1$. Let $D_{R}=\{u \in X:\|u\| \leq R\}$, then $D_{R}$ is a closed, bounded and convex set of $X$. For every $u \in D_{R}$, by means of $(\mathrm{H} 2)$ and the triangle inequality, for $t \in(0, \infty)$, we get

$$
\begin{aligned}
\| f & (s, u(s),(S u)(s),(H u)(s)) \| \\
= & \| f\left(s, \frac{1+s^{\sigma+2}}{s^{2-\alpha}} \cdot \frac{s^{2-\alpha}}{1+s^{\sigma+2}} u(s),\left(S \frac{1+s^{\sigma+2}}{s^{2-\alpha}} \cdot \frac{s^{2-\alpha}}{1+s^{\sigma+2}} u\right)(s),\right. \\
& \left.\left(H \frac{1+s^{\sigma+2}}{s^{2-\alpha}} \cdot \frac{s^{2-\alpha}}{1+s^{\sigma+2}} u\right)(s)\right) \| \\
\leq & \| f\left(s, \frac{1+s^{\sigma+2}}{s^{2-\alpha}} \cdot \frac{s^{2-\alpha}}{1+s^{\sigma+2}} u(s),\left(S \frac{1+s^{\sigma+2}}{s^{2-\alpha}} \cdot \frac{s^{2-\alpha}}{1+s^{\sigma+2}} u\right)(s),\right.
\end{aligned}
$$




$$
\begin{aligned}
& \left.\left(H \frac{1+s^{\sigma+2}}{s^{2-\alpha}} \cdot \frac{s^{2-\alpha}}{1+s^{\sigma+2}} u\right)(s)\right)-f(s, 0,0,0)\|+\| f(s, 0,0,0) \| \\
\leq & L_{1}(s) \frac{s^{2-\alpha}}{1+s^{\sigma+2}}|u|+L_{2}(s) \frac{s^{2-\alpha}}{1+s^{\sigma+2}}|S u|+L_{3}(s) \frac{s^{2-\alpha}}{1+s^{\sigma+2}}|H u|+M \\
\leq & L_{1}(s)\|u\|+L_{2}(s)\|S u\|+L_{3}(s)\|H u\|+M \\
\leq & {\left[L_{1}(s)+k_{0} L_{2}(s)+h_{0} L_{3}(s)\right]\|u\|+M \leq\left[L_{1}(s)+k_{0} L_{2}(s)+h_{0} L_{3}(s)\right] R+M, }
\end{aligned}
$$

where

$$
\begin{aligned}
& \left(S \frac{1+t^{\sigma+2}}{t^{2-\alpha}} u\right)(t)=\int_{0}^{t} K(t, s) \frac{1+s^{\sigma+2}}{s^{2-\alpha}} u(s) d s \\
& \left(H \frac{1+t^{\sigma+2}}{t^{2-\alpha}} u\right)(t)=\int_{0}^{\infty} H(t, s) \frac{1+s^{\sigma+2}}{s^{2-\alpha}} u(s) d s
\end{aligned}
$$

Now, we will show that $L D_{R} \subset D_{R}$. For all $u \in D_{R}$, by (H1), (H2), (H3) and (11), we have

$$
\begin{aligned}
\|(L u)(t)\| \leq & \sup _{t \in(0, \infty)} \frac{t^{2-\alpha}}{1+t^{\sigma+2}}\left[\int_{0}^{t} \frac{(t-s)^{\alpha-1}}{\Gamma(\alpha)}\left\{\left[L_{1}(s)+k_{0} L_{2}(s)+h_{0} L_{3}(s)\right] R+M\right\} d s\right. \\
& \left.+\frac{b}{\Gamma(\alpha)} t^{\alpha-1}+a t^{\alpha-2}\right] \\
\leq & \left\{\sup _{t \in(0, \infty)} \frac{t^{2-\alpha}}{1+t^{\sigma+2}} I_{0^{+}}^{\alpha} L_{1}(t)+k_{0} \sup _{t \in(0, \infty)} \frac{t^{2-\alpha}}{1+t^{\sigma+2}} I_{0^{+}}^{\alpha} L_{2}(t)\right. \\
& \left.+h_{0} \sup _{t \in(0, \infty)} \frac{t^{2-\alpha}}{1+t^{\sigma+2}} I_{0^{+}}^{\alpha} L_{3}(t)\right\} R+\frac{1}{\Gamma(\alpha+1)} \sup _{t \in(0, \infty)} \frac{t^{\alpha} t^{2-\alpha}}{1+t^{\sigma+2}} M \\
& +\frac{b}{\Gamma(\alpha)} \sup _{t \in(0, \infty)} \frac{t^{\alpha-1} t^{2-\alpha}}{1+t^{\sigma+2}}+a \sup _{t \in(0, \infty)} \frac{t^{\alpha-2} t^{2-\alpha}}{1+t^{\sigma+2}} \\
\leq & \left(1+k_{0}+h_{0}\right) I_{L}^{\alpha} R+\gamma_{1} M+\frac{b}{\Gamma(\alpha)} A_{1}+a A_{2} \\
\leq & \lambda_{0} R+(1-v) R \leq R .
\end{aligned}
$$

Therefore, $\|(L u)(t)\| \leq R$.

Next, we shall show that $L$ is a contraction. For $u, v \in X$ and for each $t \in(0, \infty)$, by (H1) and $(\mathrm{H} 2)$, one can get

$$
\begin{aligned}
\|(L u)(t)-(L v)(t)\| \leq & \sup _{t \in(0, \infty)} \frac{t^{2-\alpha}}{1+t^{\sigma+2}}\left[\int_{0}^{t} \frac{(t-s)^{\alpha-1}}{\Gamma(\alpha)}[f(s, u(s),(S u)(s),(H u)(s))\right. \\
& -f(t, v(t),(S v)(s),(H v)(s))] d s] \\
\leq & \sup _{t \in(0, \infty)} \frac{t^{2-\alpha}}{1+t^{\sigma+2}} \int_{0}^{t} \frac{(t-s)^{\alpha-1}}{\Gamma(\alpha)}\left\{L_{1}(s)\|u-v\|+L_{2}(t)\|S u-S v\|\right. \\
& \left.+L_{3}(s)\|H u-H v\|\right\} d s \\
\leq & \left\{\sup _{t \in(0, \infty)} \frac{t^{2-\alpha}}{1+t^{\sigma+2}} I_{0^{+}}^{\alpha} L_{1}(t)+k_{0} \sup _{t \in(0, \infty)} \frac{t^{2-\alpha}}{1+t^{\sigma+2}} I_{0^{+}}^{\alpha} L_{2}(t)\right.
\end{aligned}
$$




$$
\begin{array}{r}
\left.+h_{0} \sup _{t \in(0, \infty)} \frac{t^{2-\alpha}}{1+t^{\sigma+2}} I_{0^{+}}^{\alpha} L_{3}(t)\right\}\|u-v\| \\
\leq\left(1+k_{0}+h_{0}\right) I_{L}^{\alpha}\|u-v\|=\lambda_{0}\|u-v\|,
\end{array}
$$

where $\lambda_{0}$ is given in (H3). As $\lambda_{0}<1$, therefore, $L$ is a contraction. By the contraction mapping principle, we conclude that $L$ has a unique fixed point, which is a unique solution of problem (2).

\section{Application}

Example 3 Consider the following singular boundary value problem:

$$
\left\{\begin{aligned}
& D_{0^{+}}^{\frac{3}{2}} u(t)= \frac{1}{8\left(1+t^{\frac{3}{2}}\right)} \frac{|u|}{1+|u|}+\frac{1}{10 t^{2}(1+t)^{3}} \int_{0}^{t} \frac{s^{\frac{1}{2}} u(s)}{\left(1+s^{\frac{3}{2}}\right)(1+t+s)^{2}} d s \\
&+\frac{1}{20 t(1+t)^{3}} \int_{0}^{\infty} \frac{s^{\frac{1}{2}}}{\left(1+s^{\frac{3}{2}}\right)} e^{-s} \cos (t-s) u(s) d s, \quad t \in(0, \infty), \\
& \lim _{t \rightarrow 0} t^{\frac{1}{2}} u(t)=a, \quad \lim _{t \rightarrow 0} D_{0^{+}}^{\frac{1}{2}} u(t)=b
\end{aligned}\right.
$$

Here $\alpha=\frac{3}{2}, K(t, s)=\frac{1}{10 t^{\frac{3}{2}}(1+t)^{3}} \cdot \frac{s^{\frac{1}{2}}}{\left(1+s^{\frac{3}{2}}\right)(1+t+s)^{2}}$ and $H(t, s)=\frac{1}{20 t^{\frac{1}{2}}(1+t)^{3}} \cdot \frac{s^{\frac{1}{2}}}{1+s^{\frac{3}{2}}} e^{-s} \cos (t-s)$. Choose $\sigma=-\frac{1}{2}$. Then by direct calculations, we can obtain that

$$
k_{0}=0.1, \quad h_{0}=0.05, \quad I_{L}^{\frac{3}{2}} \approx 0.705511578 .
$$

Further, we have

$$
\lambda_{0}=\left(1+k_{0}+h_{0}\right) I_{L}^{\frac{3}{2}} \approx 0.8113383<1
$$

Then by using Theorem 2 , problem (13) has a unique solution on $(0, \infty)$.

Example 4 Consider the following singular boundary value problem:

$$
\left\{\begin{array}{l}
D_{0^{+}}^{\frac{3}{2}} u(t)=\frac{1}{3 \sqrt{\pi t}} \ln \left(2+\frac{t^{\frac{1}{2}}}{1+t^{\frac{3}{2}}} u\right), \quad t \in(0, \infty), \\
\lim _{t \rightarrow 0} t^{\frac{1}{2}} u(t)=a, \quad \lim _{t \rightarrow 0} D_{0^{+}}^{\frac{1}{2}} u(t)=b
\end{array}\right.
$$

where $a, b \in \mathbb{R}$ are constants. Here we have $\alpha=\frac{3}{2}$ and

$$
f(t, u)=\frac{1}{3 \sqrt{\pi t}} \ln \left(2+\frac{t^{\frac{1}{2}}}{1+t^{\frac{3}{2}}} u\right)
$$

Note that we have $\lim _{t \rightarrow 0^{+}} f(t, \cdot)=+\infty$. Let us choose $\sigma=-\frac{1}{2}$ and $\sigma_{0}=\frac{1}{2}, \phi(u-v)=$ $\ln (u-v+1)$. Moreover, for $u \geq v$ and $t \in(0, \infty)$, one can get

$$
0 \leq\left[\frac{1}{3 \sqrt{\pi}} \ln (2+u)-\frac{1}{3 \sqrt{\pi}} \ln (2+v)\right]
$$

since $h(x)=\ln (2+x)$ is nondecreasing on $[0,+\infty)$, and

$$
\frac{1}{3 \sqrt{\pi}}[\ln (2+u)-\ln (2+v)]=\frac{1}{3 \sqrt{\pi}} \ln \frac{2+u}{2+v} \leq \frac{1}{3 \sqrt{\pi}} \ln (u-v+1) .
$$


Also, we have

$$
\frac{\Gamma\left(\frac{3}{2}\right)}{\sup _{t \in(0, \infty)} \frac{t^{\frac{3}{2}}}{1+t^{\frac{3}{2}}} B\left(\frac{3}{2}, \frac{1}{2}\right)}=\frac{1}{\sqrt{\pi}}>\frac{1}{3 \sqrt{\pi}} .
$$

Therefore, by using Theorem 1, the boundary value problem (14) has one positive solution.

\section{Conclusions}

The existence of the positive solutions to fractional boundary value problems involving the nonlinear boundary conditions is an important issue in the area of fractional calculus, and it is a crucial step for finding the correct numerical solutions of these types of equations.

In this paper, by using a fixed point theorem in partially ordered sets and the contraction mapping principle, we have proved the existence of at least one positive solution for two problems of boundary value problems for the fractional differential equation, and we provided two illustrative examples in order to justify our approach.

\section{Competing interests}

The authors declare that they have no competing interests.

Authors' contributions

All authors have equal contributions. All authors read and approved the final manuscript.

\section{Author details}

${ }^{1}$ Department of Mathematics, Faculty of Sciences, Razi University, Kermanshah, 67149, Iran. ²Department of Chemical and Materials Engineering, Faculty of Engineering, King Abdulaziz University, P.O. Box 80204, Jeddah, 21589, Saudi Arabia. ${ }^{3}$ Department of Mathematics and Computer Sciences, Faculty of Art and Sciences, Cankaya University, Ankara, 06530, Turkey. ${ }^{4}$ Institute of Space Sciences, P.O. Box MG-23, Magurele-Bucharest, 76900, Romania. ${ }^{5}$ Department of Mathematics, Texas A M University-Kingsville, 700 University Boulevard, Kingsville, USA. ${ }^{6}$ Department of Mathematics, King Abdulaziz University, P.O. Box 80204, Jeddah, 21589, Saudi Arabia.

\section{Acknowledgements}

The authors would like to thank the anonymous referee of this paper for very helpful comments and suggestions.

Received: 17 May 2013 Accepted: 16 August 2013 Published: 8 September 2013

\section{References}

1. Hilfer, R: Applications of Fractional Calculus in Physics. World Scientific, Singapore (2000)

2. Sabatier, J, Agrawal, OP, Tenreiro Machado, JA: Advances in Fractional Calculus. Springer, Berlin (2007)

3. Baleanu, D, Diethelm, K, Scalas, E, Trujillo, JJ: Fractional Calculus Models and Numerical Methods. Series on Complexity, Nonlinearity and Chaos. World Scientific, Singapore (2012)

4. Baleanu, D, Mustafa, OG, Agarwal, RP: On the solution set for a class of sequential fractional differential equations. J. Phys. A, Math. Theor. 43(38), Article Number 385209 (2010)

5. Baleanu, D, Mohammadi, H, Rezapour, S: Some existence results on nonlinear fractional differential equations. Philos. Trans. R. Soc., A-Issue (2012, to appear)

6. Diethelm, K, Freed, AD: On the solution of nonlinear fractional order differential equations used in the modeling of viscoplasticity. In: Keil, F, Mackens, W, Voss, H, Werther, J (eds.) Scientific Computing in Chemical Engineering II Computational Fluid Dynamics, Reaction Engineering and Molecular Properties. Springer, Heidelberg (1999)

7. Mainardi, F: Fractional calculus: some basic problems in continuum and statistical mechanics. In: Carpinteri, A, Mainardi, F (eds.) Fractals and Fractional Calculus in Continuum Mechanics. Springer, New York (1997)

8. Podlubny, I: Fractional Differential Equations. Academic Press, New York (1999)

9. Kilbas, AA, Srivastava, HM, Trujillo, JJ: Theory and Applications of Fractional Differential Equations. Elsevier, Amsterdam (2006)

10. Samko, G, Kilbas, A, Marichev, O: Fractional Integrals and Derivatives: Theory and Applications. Gordon and Breach, Amsterdam (1993)

11. Liu, Y: Existence and uniqueness of solutions for a class of initial value problems of fractional differential systems on half lines. Bull. Sci. Math. (preprint)

12. Liu, Y, Ahmad, B, Agarwal, RP: Existence of solutions for a coupled system of nonlinear fractional differential equations with fractional boundary conditions on the half-line. Adv. Differ. Equ. 2013 (2013) (preprint)

13. Liang, S, Zhang, J: Existence of three positive solutions of $m$-point boundary value problems for some nonlinea fractional differential equations on an infinite interval. Comput. Math. Appl. 61, 3343-3354 (2011)

14. Liang, S, Zhang, J: Existence of multiple positive solutions for $m$-point fractional boundary value problems on an infinite interval. Math. Comput. Model. 54, 1334-1346 (2011) 
15. Zhang, L, Ahmad, B, Wang, G, Agarwal, RP: Nonlinear fractional integro-differential equations on unbounded domains in a Banach space. J. Comput. Appl. Math. 249, 51-56 (2013)

16. Harjani, J, Sadarangani, K: Fixed point theorems for weakly contractive mappings in partially ordered sets. Nonlinear Anal. 71, 3403-3410 (2009)

17. Caballero Mena, J, Harjani, J, Sadarangani, K: Existence and uniqueness of positive and nondecreasing solutions for a class of singular fractional boundary value problems. Bound. Value Probl. 2009, 3403-3410 (2009)

doi:10.1186/1687-1847-2013-266

Cite this article as: Nyamoradi et al.: Existence and uniqueness of positive solutions to fractional boundary value problems with nonlinear boundary conditions. Advances in Difference Equations 2013 2013:266.

Submit your manuscript to a SpringerOpen ${ }^{\circ}$ journal and benefit from:

- Convenient online submission

- Rigorous peer review

- Immediate publication on acceptance

- Open access: articles freely available online

- High visibility within the field

- Retaining the copyright to your article

Submit your next manuscript at $\gg$ springeropen.com 